\title{
Model of coexistence of annual plants in a heterogeneous habitat
}

\author{
Michel Droz ${ }^{1}$ and Andrzej Pękalskii ${ }^{*}$ \\ ${ }^{1}$ Department of Theoretical Physics, University of Genèva, 1211 Genève 4, Switzerland \\ ${ }^{2}$ Institute of Theoretical Physics, University of Wrocław, pl. M. Borna 9, 50-203 Wrocław, Poland
}

Article history

Received: 20 December 2017

Accepted: 29 March 2018

Published: 03 April 2018

(C) Droz and Pękalski (2018)

Editor

K K Sabu

\section{Publisher}

Horizon e-Publishing Group

Correspondence

Andrzej Pękalski

$凶$ andrzej.pekalski@ift.uni.wroc.pl

\begin{abstract}
We propose a computer simulation type model describing dynamics of a system of annual plants competing for just one resource and living in a heterogeneous habitat. Plants do interact via blocking a part of the resource in the nearest neighbourhood. Species differ in only one aspect - demand for the resource. Plant which has supply equal demand has the largest probability to survive, while any deviation diminishes it. Heterogeneity in space is introduced in two ways - by a gradient reducing supply along one axis and a system of patches, each having a different level of the resource. We show that without any trade-off mechanism, speciation or immigrants, coexistence of species is possible in a stationary state. We find out also that the two descriptions of the heterogeneity lead to nearly the same numbers of surviving species, although the spatial structures and order of abundance of the populations are different.
\end{abstract}

\section{Keywords}

plant dynamics; Monte Carlo simulations; water supply-demand mismatch; annual plants; spatial heterogeneity

\section{Citation}

Droz M, Pękalski. Model of coexistence of annual plants in a heterogeneous habitat. Plant Science Today 2018;5(2):55-63. https://dx.doi.org/10.14719/pst.2018.5.2.369

\section{Introduction}

One of the important problems in ecology is the coexistence of species (1). Many mechanisms have been proposed to explain it - competitioncolonisation trade-off (2-7), different kinds of disturbance, like flooding, grazing or fire $(8,9)$, as well as changes of the habitat in time $(10,11)$ or heterogeneity in space $(1,3,12,13)$. Maintaining biodiversity is possible, according to the neutral theory of Hubbell (14) via stochastic processes and speciation. A model exploring this kind of dynamics has been proposed in (13).
In our previous paper (15) we have presented a model of annual plants living in a homogeneous habitat. We have shown that coexistence is possible, without any kind of a trade-off, speciation or immigrants, although it has a transitory character and can be realised in a rather narrow range of the resource supply. In the present paper we study the system of annual plants, also differing in only one aspect and living in a heterogeneous habitat.

Different types of spatial heterogeneity (SH) are encountered in nature (16), but for modelling it two approaches are generally used. In the first one a 
quasi-continuous gradient in one or more resources $(10,17,18)$ is the source of the SH. In the second one $(3,8,19)$ a number of patches on which the resource has different values, is introduced. The problem of defining heterogeneity is a difficult one, as shown in (16) and it is important in theoretical and simulation models in view of the recently very active (2022) topic of the relation between $\mathrm{SH}$ and biodiversity (BD). To the best of our knowledge it has never been checked whether the two approaches are equivalent in all or only in some special conditions. Without such a check, statements coming from the results of one type of description may have a rather limited validity.

We do not assume in our model any kind of a trade-off mechanism, meaning that plants which are less fit to a given condition can not compensate it by, for example, larger fecundity (7). In particular we want to determine the influence on biodiversity of the following factors, which a priori could play an important role:

\section{Number of competing species,}

2. Degree of heterogeneity, and

3. Type of heterogeneity (gradient or patches)

We also want to find out how the composition of the total population (all species) and dominance of species change in time, as a function of the above factors. Dominance means here that one species is more abundant than the other (23).

\section{Model}

The habitat we investigate is a square lattice of size $L \times L$ with hard boundary conditions - nothing can get outside of the system. On each cell of the lattice can grow only one plant, or the cell may be empty. Plants are competing for a single resource (water) and are characterised by the demand for it, which is identical for all plants belonging to a given species. Since the plants are using the same resource, we have here the case of complete niche overlap, as defined by Chesson (1). All quantities related to water are in the same, arbitrary, units. Apart from different demands for water, the species are identical. There is no compensation coming from other factors for species which are worse fit to actual conditions. There is no speciation nor immigrants.

Life cycle of the plants is composed of two phases - germination, when the seeds germinate, and adult, when the plants produce seeds, disperse them and then die. In the adult phase each plant competes with its four nearest neighbours (NN) for water. Namely, a part of water nominally available to a plant (rainfall on the cell) is blocked by roots of these NN (24) and therefore effectively a plant located at the cell with coordinates $(x, y)$ receives the amount of water

$$
v_{i}(x, y)=w(x, y)\left(1-0.1 n n_{i}\right),
$$

where $w(x, y)$ is the amount of rainfall on the cell $(x, y), n n_{i}$ is the number of NN the plant $i$ has and the factor 0.1 characterisers the strength of the interactions among plants and is a compromise between a larger value, for which the effect of water will be lost, and a smaller value when the plants will not feel their presence.

In our model a departure from the optimum supply, equal the demand, has always a negative effect on the plant. In biological literature there is no standard form describing the chance a plant has in surviving the given external conditions. We propose the following simple form, which is of a Gaussian type (13) and satisfies the natural criteria - it peaks when the supply equals the demand, goes to zero if there is no water or the supply is very large. Hence, the chance of survival for a plant $i$ of species $m$, with the demand $d(m)$, located at cell $(x, y)$ is defined as

$$
p_{i}(m ; x, y)=\exp \left(-\left[v_{i}(x, y)-d(m) / v_{i}(x, y)\right]^{2}\right) .
$$

A random number $r_{i} \in(0,1)$ is taken from a uniform distribution. If it is larger than $p_{i}(m ; x, y)$, the plant dies and is removed from the system. Otherwise it survived and produces seeds, which number depends on $p_{i}(m ; x, y)(25)$

$$
s_{i}(m ; x, y)=E\left[6 p_{i}(m ; x, y)\right],
$$

where $E[. .$.$] means the integer part of [ .. ]. 6$ is the maximum number of seeds a plant can produce in optimal conditions. The seeds are distributed randomly over 13 sites in the neighbourhood of the plant. The choice of 13 cells is a compromise between putting them in the nearest neighbourhood and spreading over the whole habitat.

In the germination phase each cell is visited just once and in a random order. From the cell one seed is chosen for germination and put to the germination test, which is analogous to eq.(2), except that seedlings do not block water. Therefore instead of $v_{i}(x, y), w(x, y)$ is used. When on a cell there are seeds of different types, the one for germination is chosen following the majority rule (27). Namely, if there are $s_{1}$ seeds of plants of type 1 and $s_{2}$ seeds of plants of type 2, the probability of choosing a seed of type 1 is $s_{1} /\left(s_{1}+\right.$ $s_{2}$ ). It would be analogously for a larger number of species.

The rainfall in our habitat is not uniform in space, as we have assumed before (15), but is heterogeneous. Each year however the amount falling on a given cell is the same. 

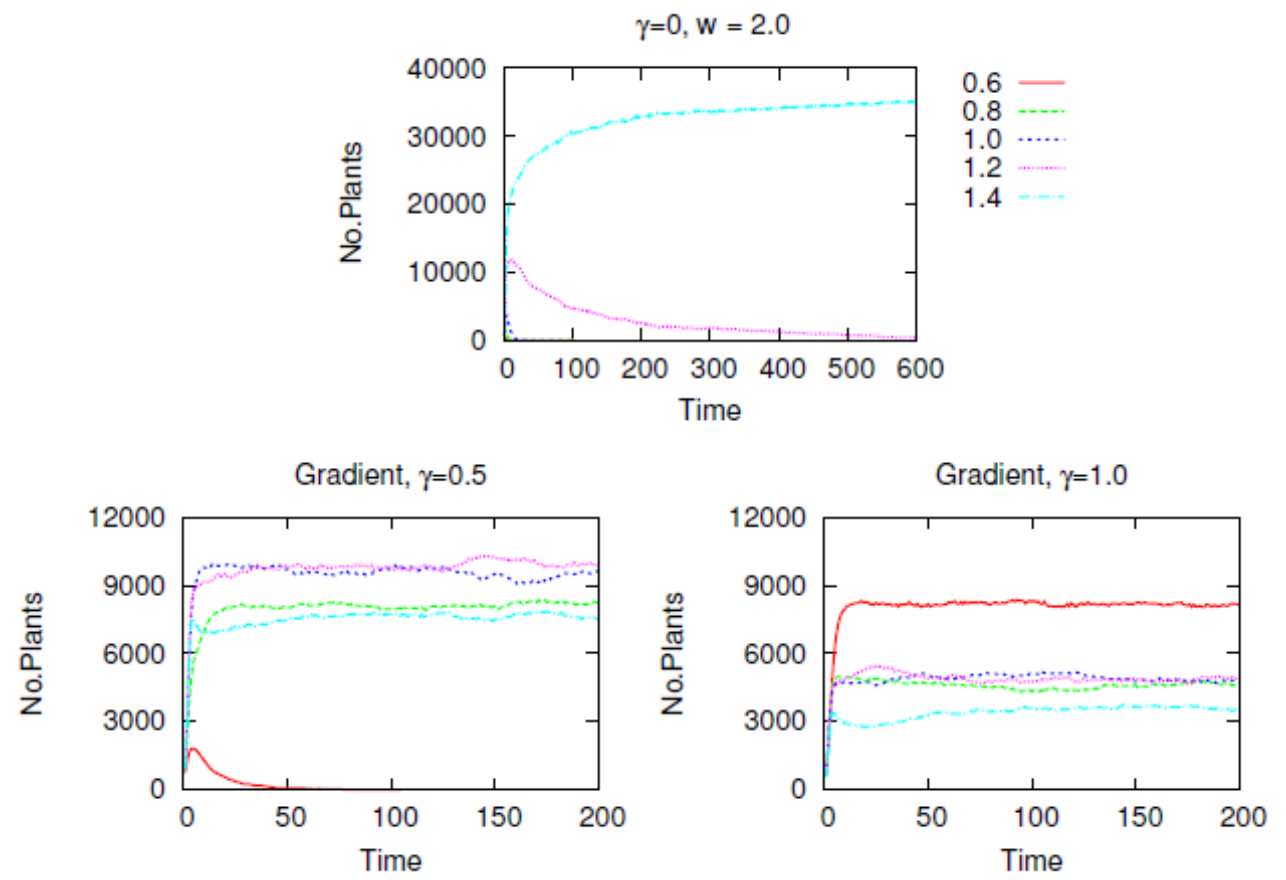

Fig 1. Time development of plant abundances. Homogeneous and gradient habitat. Note different scales
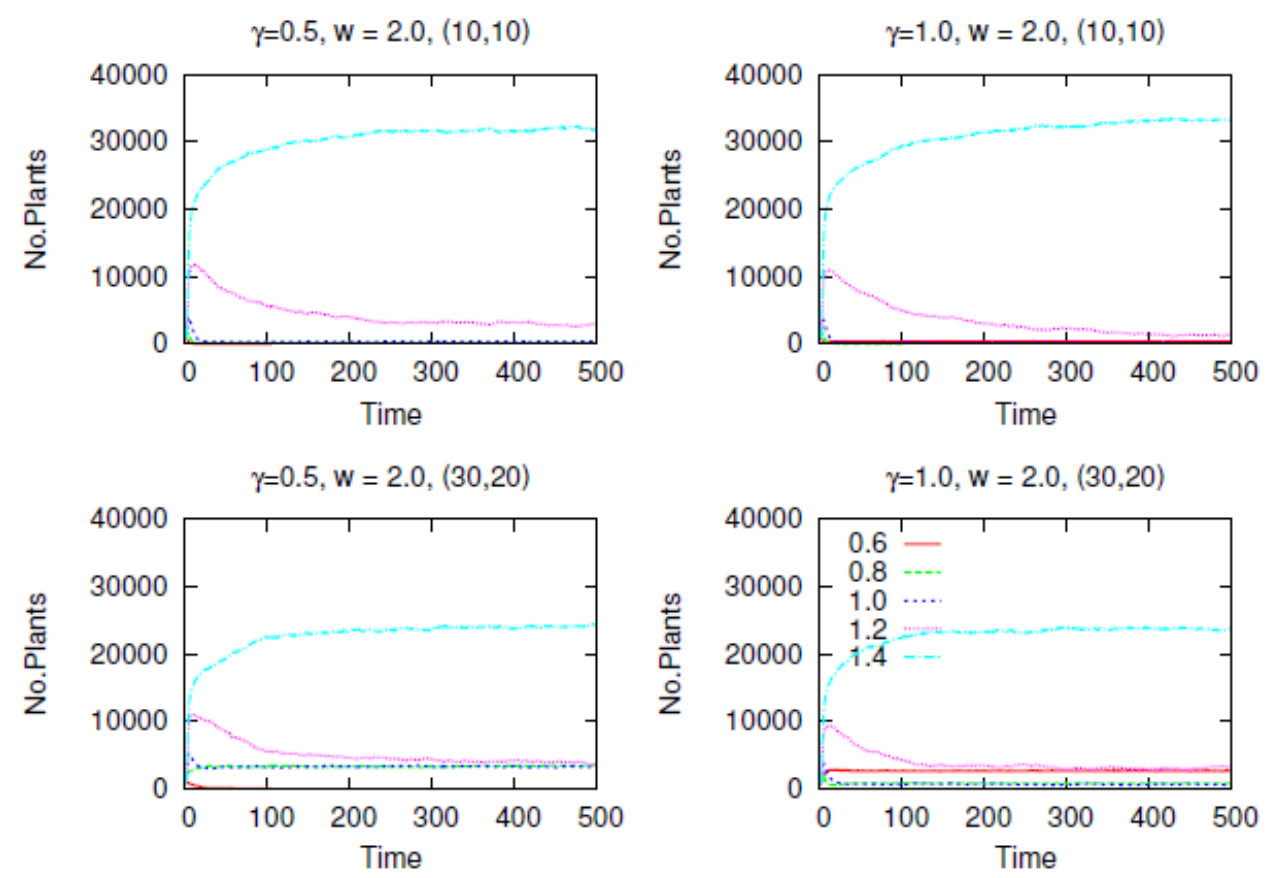

Fig 2. Time development of plant abundances for patchy systems

There are basically two methods used to describe spatial heterogeneity. The first type is introduced as a quasi- continuous gradient $(10,17$, 18) of steepness $\gamma$ along the $O X$ axis when the moisture decreases with increasing $x$ coordinate as

$$
w g(x)=w\left(1-\frac{\gamma \cdot x}{L}\right)
$$

where $w$ is the maximum value of the rainfall (our control parameter). The value of the $y$ coordinate is immaterial since all cells for a given value $x$ have the same moisture. The gradient is quasi- continuous because the $x$ variable changes in a discrete way. With increasing value of $\gamma$ the habitat becomes more diversified, hence we assume that the value of $\gamma$ is our measure of $\mathrm{SH}$. The total amount of water the system receives, depends on the value of the gradient and is equal to $L^{2} w(1-0.5 \cdot \gamma)$.

Another type of SH $(3,8,19)$ is introduced by putting on the lattice a certain amount, $n$, of square patches of the same size $l \times l$ with attributed randomly different values of the 

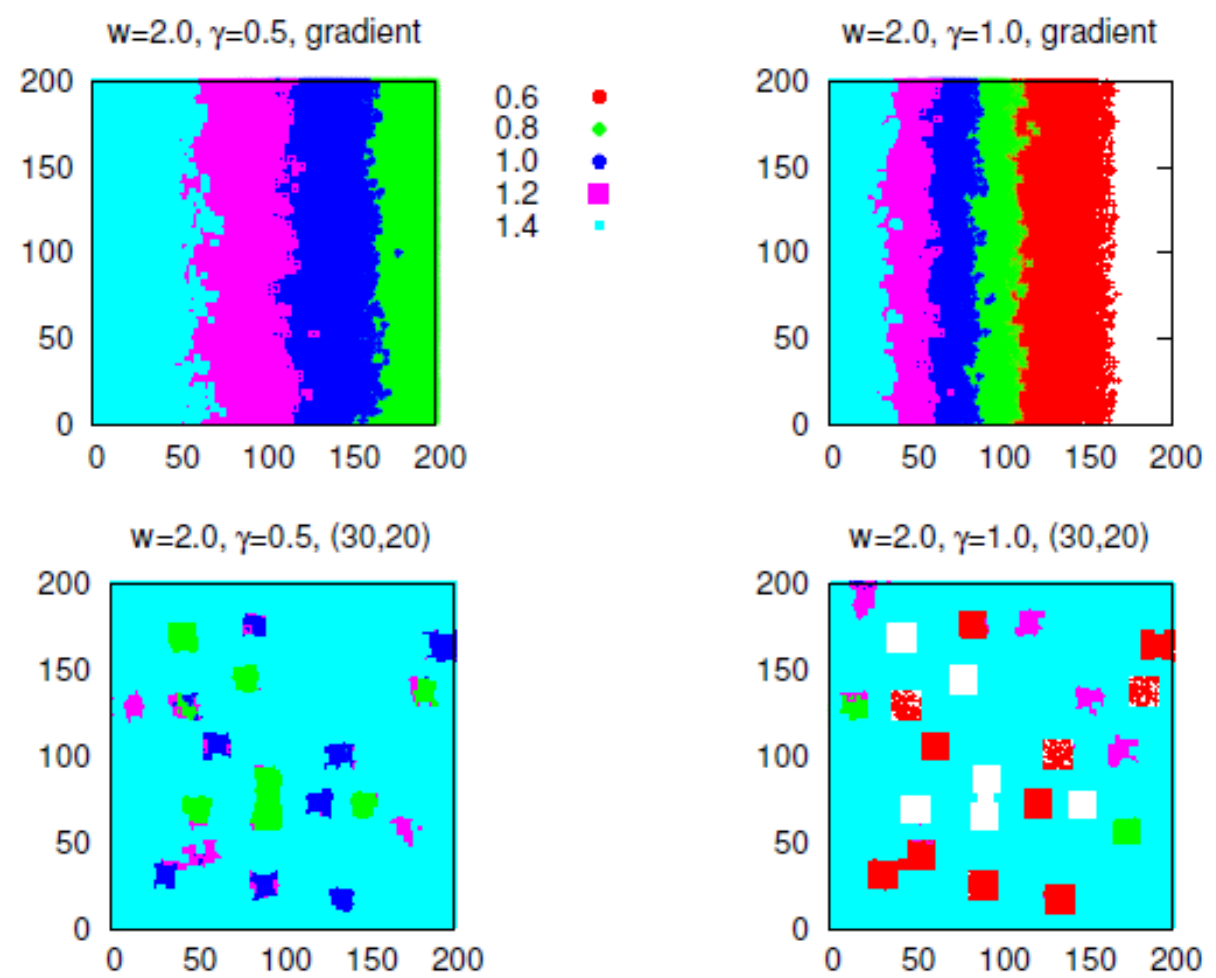

Fig 3. Spatial arrangements of plants after 200 years for the gradient case (upper panels) and for patches (bottom panels). Colours correspond to those in the previous figures. White regions indicate empty space

moisture on each patch. Outside of the patches the moisture is $w$. The patches are distributed randomly over the lattice. They can touch each other, but cannot overlap. All cells within a given patch have the same amount of water. In order to have correspondence with the case of the gradient, we assume that for a given gradient steepness $\gamma$ the amount of water on patches may vary in the same range as for the gradient case, namely between $w$ and $w(1-\gamma)$. The difference is proportional to the gradient steepness and a cell having, for example, its right top corner at $(x, y)$ has the water given by

$$
w p(x, y)=w(1-\gamma)+r_{x y} \gamma \cdot w,
$$

where $r_{x y}$ is another random number.

\section{Results}

The simulations were carried out on a lattice of $200 \times 200$ cells. Initially 1000 plants of each type were put on the lattice in randomly chosen cells. We have the following parameters with fixed value: size of the system, initial number of plants of each type. maximum number of seeds a plant can produce in a year, time of simulations. Our control parameter are the steepness of the gradient $\gamma$, which also determines the level of heterogeneity and the water supply $w$. Size and number of patches are also our control parameters. How the results change when we take another values for the parameters with fixed values, hence the robustness of the model, will be discussed in the next section.

\subsection{Five types of plant}

We start with the system of 5 types of plant $(i=1, .$. 5) having the demands $-d_{i}=0.6,0.8,1.0,1.2$ and 1.4, respectively. In Fig. 1 the time dependence of the number of plants of the five types is presented for a water supply $w=2.0$. The curves represent history of one population (there is no averaging). Populations of the same species but initially located at different positions have quite similar history. The upper panel shows the case of a homogeneous habitat, while on the bottom panels the habitat is heterogeneous with a medium $(\gamma=$ $0.5)$ and strong $(\gamma=1.0)$ heterogeneity of the gradient type. As seen from the upper panel, homogeneous habitat can support just one species, best fitted to the conditions (26). In this case it is the species with highest demand for water. When the habitat becomes heterogeneous, more species can exist, as seen from the bottom panels. Moreover, the system stabilises, reaching a stationary state. When the gradient is large $(\gamma=$ 1.0) the species with the highest demand for water is no longer the dominant one, but its place is taken by the species with the lowest demand. This change of dominance is explained by inspection of upper panels in Fig. 3. White region at the rightmost part of the top right panel $(\gamma=1.0)$ shows areas with not enough of water to sustain any kind of plant. In a large part of the habitat 

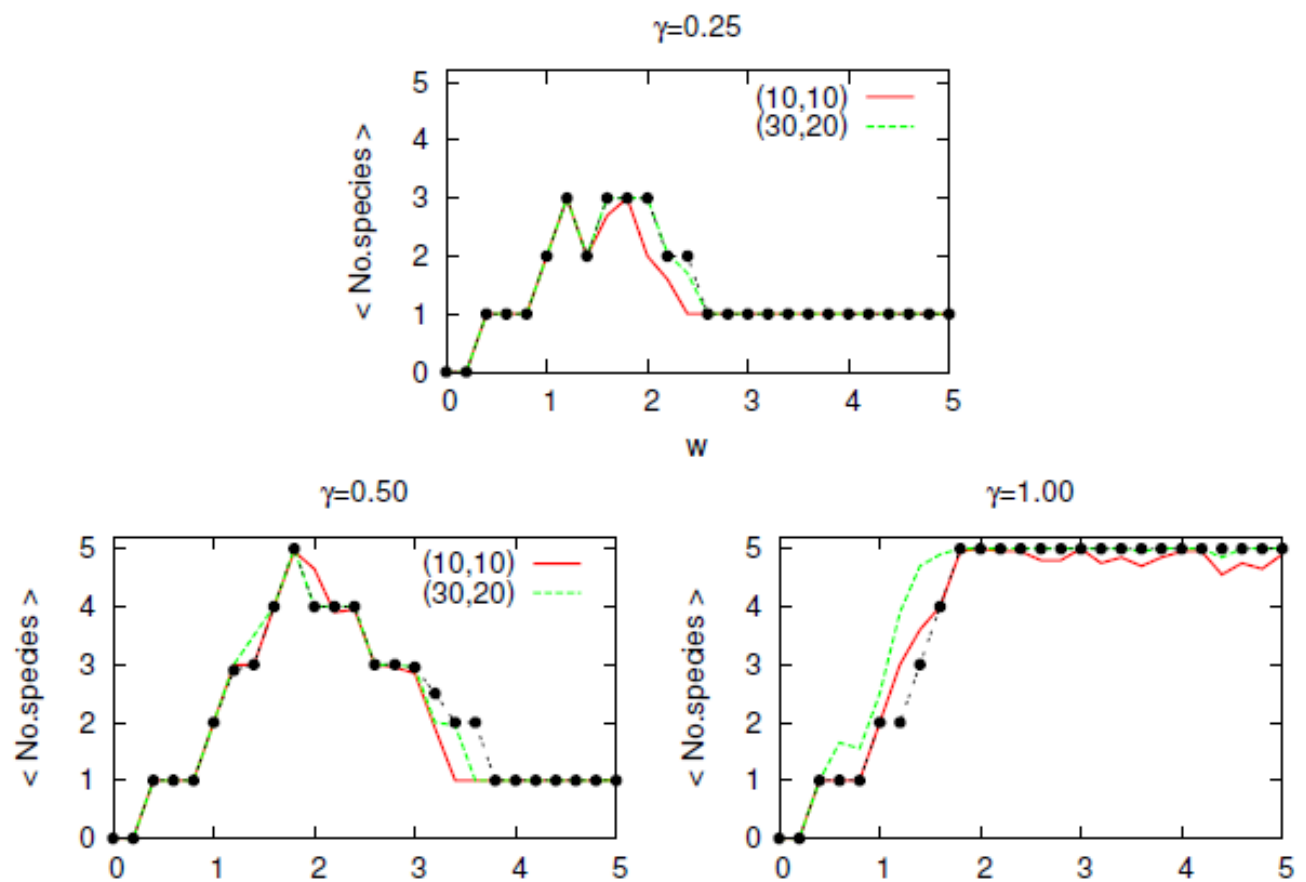

Fig 4. Average number of surviving species versus water supply. Gradient case is represented by black dots

water is scarce, hence only plants with small demand for it can flourish.

In Fig. 2 time dependence of plants' abundances are shown for two patchy systems with 10 patches of size $10 \times 10$, labelled $(10,10)$ and for 30 patches of size $20 \times 20$, labelled $(30,20)$, each for two values of SH $-\gamma=0.5$ and $\gamma=1.0$. The former system offers slightly worse conditions for $\mathrm{BD}$ as more species can survive in the $(30,20)$ system. Here however, in contrast to the gradient case, always the species with the highest demand is the dominant one. Again, explanation can be found in Fig. 3. Even in the $(30,20)$ system patches cover only a part of the habitat. In the remaining part conditions are like in the homogeneous case, hence the plants with the highest demand are privileged. Since part of the rainfall is blocked by NN water supply $w=2.0$ may by optimal for plants with the demand equal 1.4.

It should be noticed that the presented figures were obtained for a given value of the water supply $w=2.0$. Would we decide to choose another value of $\mathrm{w}$, a different type of plant could be dominant. How the population of 5 types of annual plants behaves for different values of the water supply, is presented in Fig. 4 . The data were obtained for simulations lasting till 200 years. Averaging was over 20 runs.

When the SH is rather low $(\gamma=0.25)$ only up to 3 species can exist. When the $\mathrm{SH}$ increases to $\gamma=0.5$ this number reaches the maximum 5 for one special value of the water supply and extends over a large region of water supply when $\mathrm{SH}$ is high $(\gamma=1.0)$. There is practically no difference in the way the SH is modelled - by a gradient or the two patchy systems. Although the average number of surviving species does not depend strongly on the way SH is modelled (see Fig. 4), the structure depends on the type of modelling. Gradient-type heterogeneity reduces dominance of one type of species by offering each species the same amount of space. Small patches $(10,10)$ do not give equal opportunity to each species since in a large part of the habitat the moisture is equal to the rainfall $\mathrm{w}$ and plants with similar demand are privileged, as seen in Fig. 5. When the number and size of the patches increases, the picture becomes more similar to the gradient case. How the abundances of each species, taken after 200 years, behave when the water supply is changed, is shown in Fig. 5 for the gradient and small patches cases. In general two species - with the lowest and highest demand for water are most numerous, for the low and high water supply respectively. In the intermediary zone of water supply more types of plant can exist, hence the abundances of each type are lower than those at the extremes.

The large zones of existence of single species with the highest demand for water, shown in Fig. 4 for weak SH $(\gamma=0.25)$ can be explained as follows. Let us define tolerance of species with water demand $d$ as the range of water supply $v$, for which the survival chance, defined by eq.(2) is larger than a given value $p$. Since the same form is used to determine the germination success and plant's survival, the value of $p$ should be larger than 0.5. As both, lack and surplus of water have negative effect on the plants, we have two limiting values for the water supply. Plants with a given demand $d$ may survive if the water supply is between the corresponding values on the upper and lower lines. The dependence of tolerance defined in this way, on the demand for water, is shown in Fig. 6. We see from it that the tolerance 
$(10,10), \gamma=0.5$

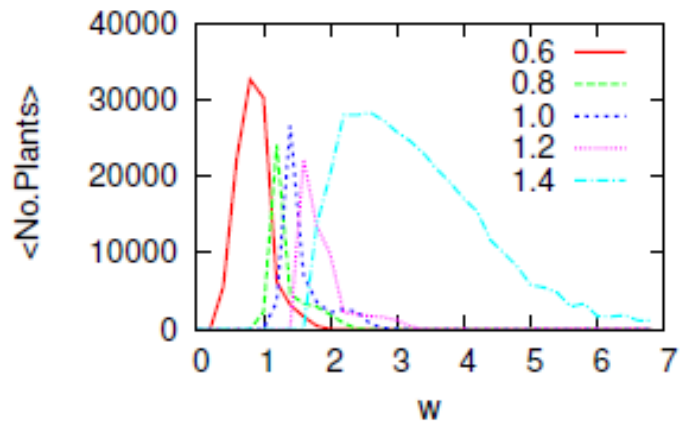

Gradient, $\gamma=0.5$

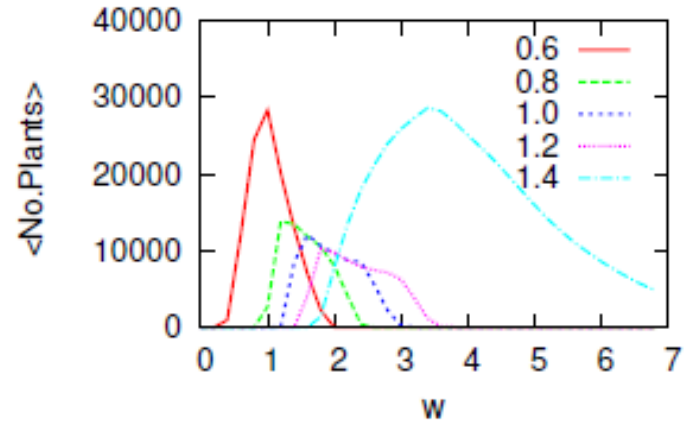

$(10,10), \gamma=1.0$

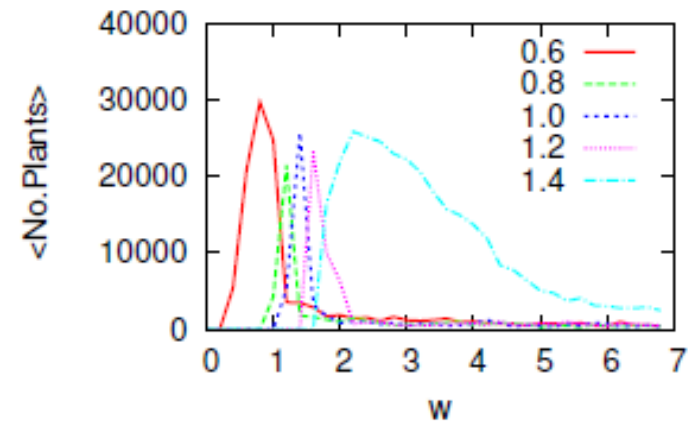

Gradient, $\gamma=1.0$

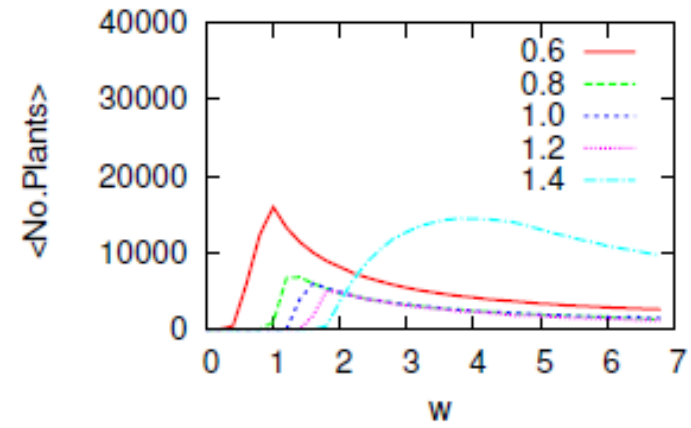

Fig 5. Abundances of 5 types of plant vs water supply for the $(10,10)$ patches (top panels) and the gradient case (bottom panel). Medium and strong SH. Legend shows plants' demand for water

increases with the demand for water. This means that such plants can exist in a wider region of water supply than plants having small demand for water. We have been unable to find a comment on this relationship in the literature.

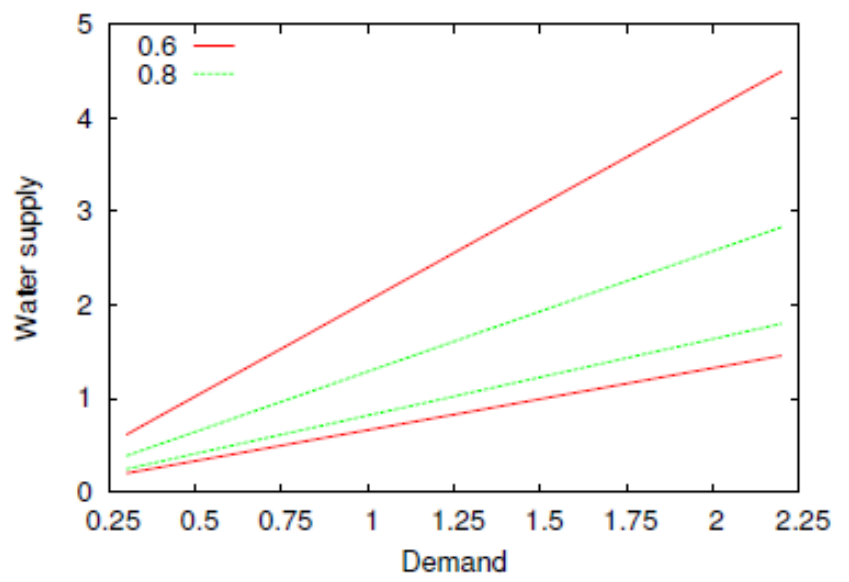

Fig. 6. Upper and lower limits for the tolerance. For a given value of the demand plants can surive with probability 0.6 for water supply between the red lines and with probability 0.8 for w between green lines

\subsection{Fifteen species}

So far we have considered the case of just 5 species differing in their demand for water by 0.2 . How the situation looks when we have more species on the same habitat and differing by just 0.1 (in the range [0.4 - 1.9]), is shown in Fig. 7 for the case of 15 species the homogeneous habitat and gradienttype heterogeneity. In Fig. 8 the same dependence for two patch $(30,20)$ systems and the same level of SH as in Fig. 7, is shown. For smaller patches $(10,10)$ the two dominant species with high demand ( $d=1.2$ and $d=1.4$ ) remain most numerous. Other species, which have abundances about 2000 in the case of $(30,20)$ patches, are reduced to about 200 for the $(10,10)$ patches.

In Fig. 9 the average number of species surviving till 500 years as a function of water supply $w$ is shown for a system with 15 species. The picture is not much different from that for 5 plants (see Fig. 4), except that now a larger degree of SH is needed to support all species. The peaks of the curves are also shifted to higher values of $\mathrm{w}$, as now more species is competing for water and some of them have higher demand for water than the most "thirsty" ones among 5 species. The data are averaged over 50 independent realisations.

Although the gradient and $(10,10)$ systems yield nearly the same average number of existing species, the structure of the populations is different as can be seen from the time development curves. The $(10,10)$ system offers smaller SH than the gradient system and therefore, specially for large water supply, the dominance of one species is much stronger in the patchy system.

\section{Robustness of the model}

The form of the survival chance (eq.(2)) follows the general trends in biology to use Gaussian-like types, but our results remain qualitatively similar if we take another, also non-Gaussian, forms of the survival chance. 

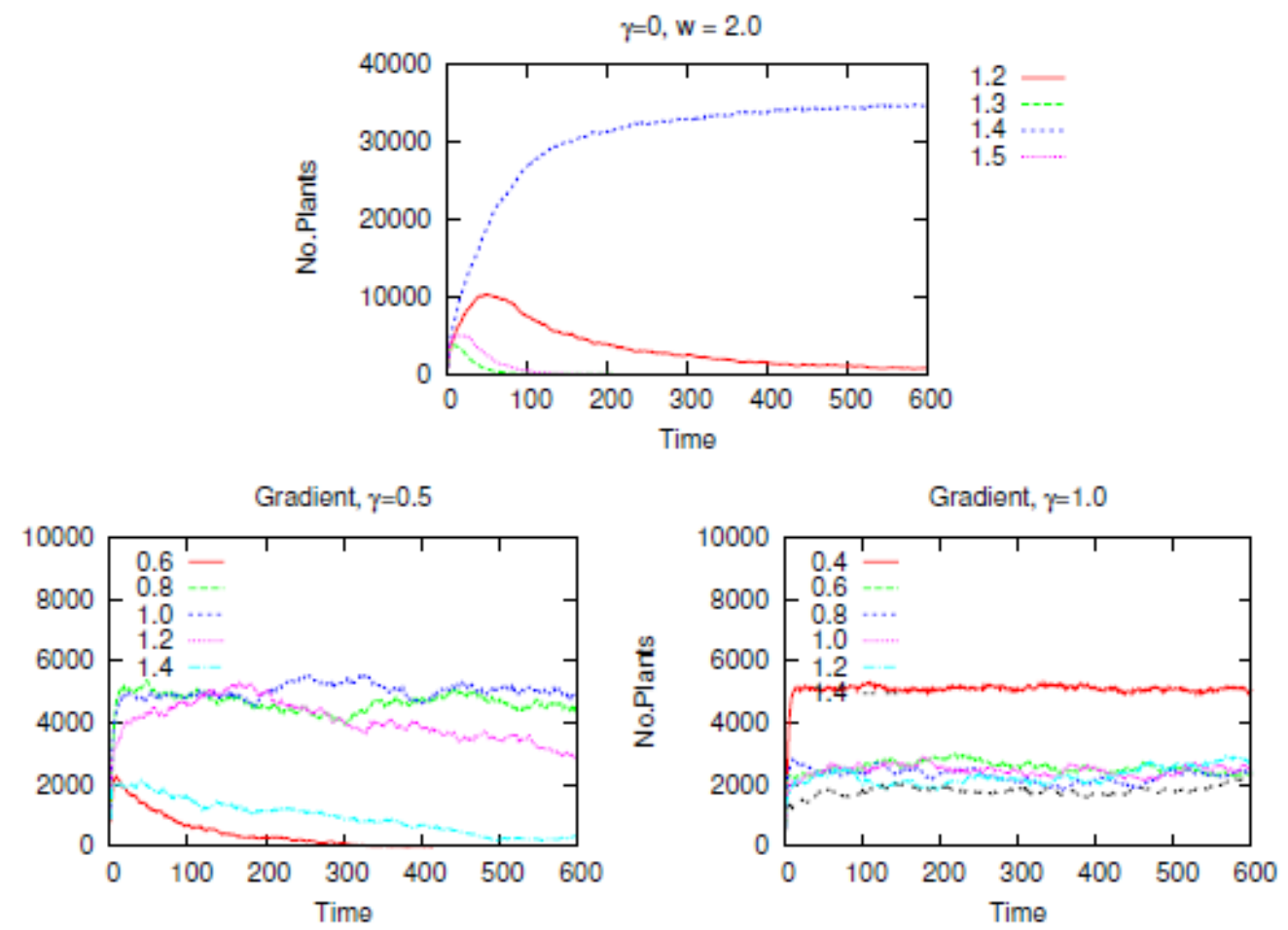

Fig. 7. Time dependence of plants abundances for the homegeneous habitat (top panel) and gradient for 15 species. Species which disappeared fast are not shown. For clarity only every second of surviving species is presented. Note different scales
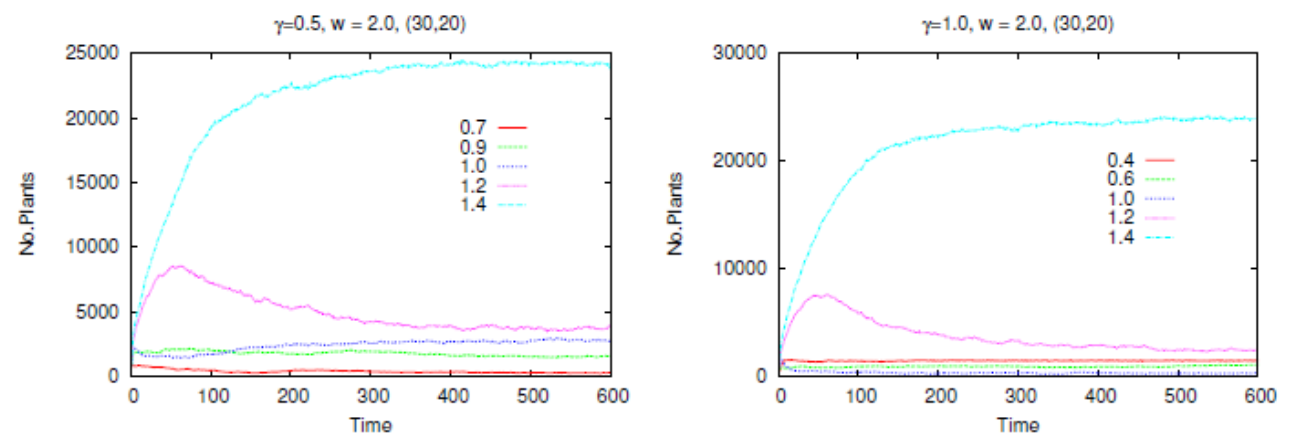

Fig. 8. Time dependence of plants abundances for patchy systems (15 species)
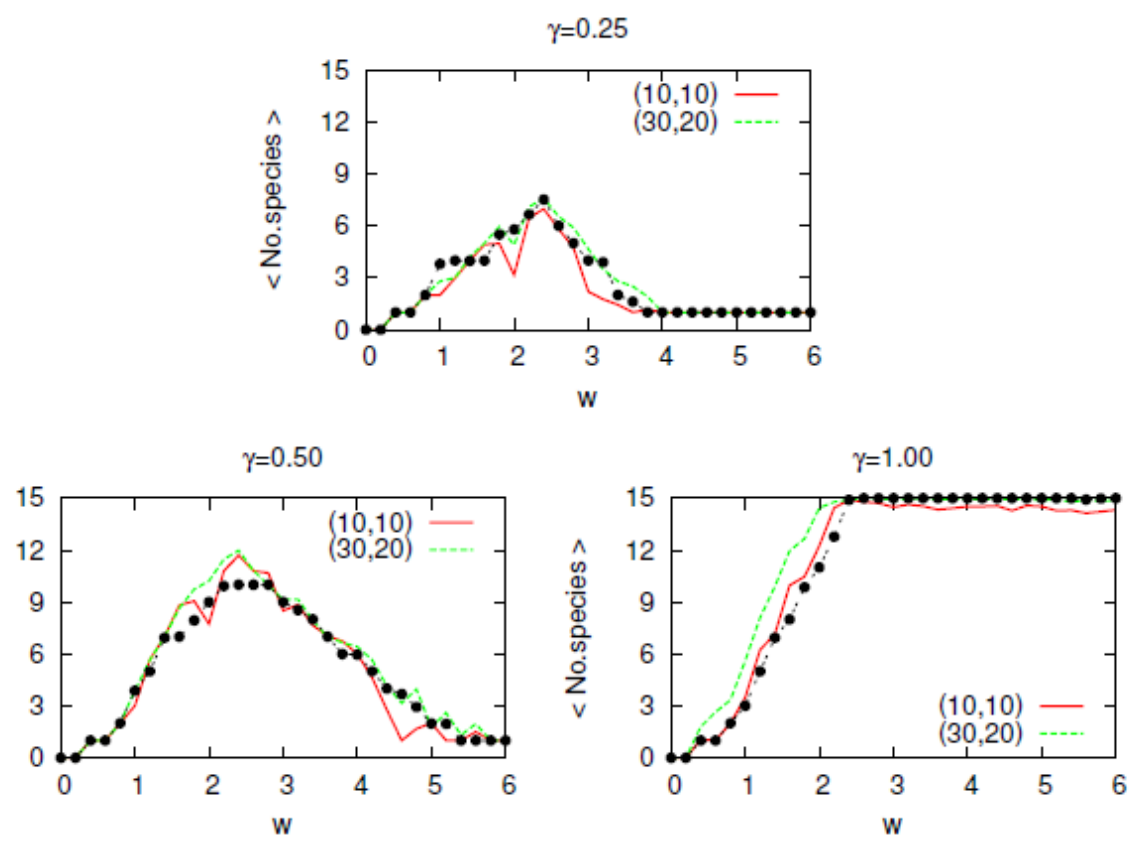

Fig. 9. Average number of species (total 15) at the end of simulations (500 years) Black dots reperent data for the gradient-type SH. Average over 50 runs 
The size of the habitat is not an important parameter, as long as it is not very small, where stochastic effects play a role $(1,26)$. Similar effect has reduction of the initial number of plants.

As seen from Fig. 1 and 2, in the heterogeneous habitat the plant abundances stabilise rather fast and after 200 years (plants' life cycles) the system is in a stationary state. Extending further the simulation has no effect on a population of 5 species. With a larger number of species stationary state is attained a little later. The results for 15 species were obtained from simulations lasting till 500 years.

Since from a cell only one seed is taken for germination test, the maximum number of seeds a plant can produce in a year, is not a relevant parameter. Again, as long as it is not as low as 1 or 2 , in which case the system will be driven to extinction.

We have considered here a system of patches distributed randomly over the lattice. If we however divide the lattice into identical squares, the results will be very similar for the average number of species surviving as a function of water supply. Also the way water is distributed over the patches does not play an important role. In our model each patch receives a random amount of water taken from the range [(1- $-\gamma)$ $w, w]$. Another method is to divide this range into as many segments as there are patches and then attribute to a patch randomly selected value taken from this set. The results remain the same in the two approaches.

\section{Conclusions and discussion}

We have presented and discussed a model of annual plants living in a habitat with heterogeneous distribution of the only resourcewater. The plants are suffering from both - lack or too much of water. The species differ only by their demand for it. Otherwise they are identical. To the best of our knowledge there is no study which covers the same problems - coexistence of different species of annual plants in a heterogeneous habitat and differing just by one feature, without any kind of a trade-off, immigrants or speciation. There was also no comparison of the two descriptions of $\mathrm{SH}$ - via gradient or patches and the equivalence of them has never been established.

We have shown that, contrary to the case of a homogeneous habitat where in analogous populations of annual plants coexistence is a transitory phenomenon (15), in a heterogeneous system species coexist in a stationary state, as they are able to find niches with water conditions well suited to their demands.

The range of water supply over which all types of plants can exist depends on the number of species and SH. As could be expected, larger number of competing species require more diversified habitat. When heterogeneity is very strong $(y=1.0)$, all types of plants can exist and moreover for a wide range of water supply.

There is no qualitative difference between the behaviour of a system of 5, 15 or 30 species. The way SH is modelled - via a gradient or patchy system is non relevant as long as only the number of surviving species is considered. Both approaches yield very similar results for the average number of surviving species, although the spatial distribution is quite different. Also the dominant species are not the same in the two approaches.

The two factors which determine the fate of the population composed of several species of annual plants are the water supply and SH. Changing just those two parameters is enough to build a system exhibiting a stationary state with many coexisting species.

Our results do not contradict the competitive exclusion principle, known also as the Gause law (28) which states that coexistence of species competing for the same resource is impossible. In our case the habitat is heterogeneous, hence it offers different conditions for species, allowing coexistence.

It would be interesting to develop further the model presented above and include factors which were deliberately left off here. These could be - competition for more than one resource, some kind of a trade-off, temporal changes of the habitat or mechanisms replacing the simple majority rule for choosing a seed for germination.

\section{Conflict of interest}

The authors declare no conflict of interest.

\section{Authors' contribution}

MD performed the review of literature. AP did the simulations. The text was written in close collaboration.

\section{References}

1 Chesson P. Mechanisms of maintenance of species diversity. Annual Review of Ecology and Systematics 2000; no. 31, pp. 343-366.

2 Levins R, Culver D. Regional coexistence of species and competition between rare species. Proceedings of the National Academy of Sciences 1971;6:12461248.

3 Tilman D. Competition and biodiversity in spatially structured habitats. Ecology 1994;75:2-16. doi: $10.2307 / 1939377$

4 Holmes E, Wilson H. Running from trouble: long distance dispersal and the competitive coexistence of inferior species. American Naturalist 1998;151:578-586. doi:10.1086/286143 
5 Yu D, Wilson $\mathrm{H}$. The competition-colonization tradeoff is dead; long live the competition-colonization trade-off. The American Naturalist 2001;158:49-63.

6 Coomes D, Rees M, Turnbull L, Ratcliffe S. On the mechanisms of coexistence among annual-plant species, using neighborhood techniques and simulation models. Plant Ecology 2002;163:23-39. doi:10.1086/286143

7 Muller-Landau H C. The tolerance-fecundity tradeoff and the maintenance of diversity in seed size. Proceedings of the National Academy of Sciences 2010;107:4242-4247.

8 Roxburgh S, Shea K, Wilson J. The intermediate disturbance hypothesis: patch dynamics and mechanisms of species coexistence. Ecology 2004;85:359-371. doi:1890/03-0266

9 Miller A, Reilly D, Bauman S, Shea K. Interactions between frequency and size of disturbance affect competitive outcomes. Ecological Research 2012;27:783-791. doi: 10.1007/s11284-012-054-4

10 O’Brien E. Climatic gradients in woody plant species richness: towards an explanation based on an analysis of southern Africa's woody flora. Journal of Biogeography 1993;181-198.

11 Seifan M, Seifan T, Jeltsch F, Tielborger K. Combined disturbances and the role of their spatial and temporal properties in shaping community structure. Perspectives in Plant Ecology, Evolution and Systematics 2012; 14:217-229.

12 Pacala S. Neighborhood models of plant population dynamics. 2. multi-species models of annuals. Theoretical Population Biology 1986;29:262-292.

13 Schwilk D W, Ackerly D D. Limiting similarity and functional diversity along environmental gradients. Ecology Letters 2005;8:272-281. doi:10.1111/j.14610248.2004.00720x

14 Hubbell S P. The unified theory of biodiversity and biogeography. Princeton University Press, Princeton, NJ; 2001.

15 Droz M, Pękalski A. Role of tolerance to resource demand-supply mismatch in a model of annual plants. Plant Science Today 2017;4:191-201. doi:10.14719/pst.2017.4.4332

16 Stein A, Gerstner K, Kreft H. Environmental heterogeneity as a universal driver of species richness across taxa, biomes and spatial scales. Ecology Letters 2014;17:866-880. doi:10.1111/ele.12277
17 Wilson W, Nisbet R. Cooperation and competition along smooth environmental gradients. Ecology 1997;78:2004- 2017.

18 Travis J, Brooker R, Clark E, Dytham C. The distribution of positive and negative species interactions across environmental gradients on a dual-lattice model. Journal of Theoretical Biology 2006;241:896-902.

19 Silvertown J. Plant coexistence and the niche. Trends in Ecology \& Evolution 2004;19:605-611.

20 Kadmon R, Allouche O. Integrating the effects of area, isolation, and habitat heterogeneity on species diversity: a unification of island biogeography and niche theory. The American Naturalist 2007;170:443-454.

21 Hortal J, Triantis K, Meiri S, Thèbault E, Sfenthourakis S. Island species richness increases with habitat diversity. The American Naturalist 2009;174:E205-E217.

22 Lundholm J. Plant species diversity and environmental heterogeneity: spatial scale and competing hypotheses. Journal of Vegetation Science 2009;20:377-391.

23 Connolly J, Wayne P, Bazzaz F. Interspecific competition in plants: how well do current methods answer fundamental questions? American Naturalist 2001;157:107-125. doi: $10.1086 / 318631$

24 Schenk H. Root competition: beyond resource depletion. Journal of Ecology2006;94:725-739. doi: 10.111/j.1365-2745.2006.01124.x

25 Shirley $\mathrm{H}$. The influence of light intensity and light quality upon the growth of plants. American Journal of Botany 1929;16:354-390.

26 Levin S. Community equilibria and stability, and an extension of the competitive exclusion principle. American Naturalist 1970;104:413-423. doi:10.1086/28.2676

27 Chesson P, Warner R. Environmental variability promotes coexistence in lottery competitive systems. American Naturalist 1981;117:923-943.

28 Hardin G. The competitive exclusion principle. Science doi:10.1126/science.131.34098.1292 